\title{
An exploration of information exchange by adolescents and parents participating in adolescent idiopathic scoliosis online support groups
}

Traci Schwieger ${ }^{1 *}$, Shelly Campo ${ }^{2}$, Keli R. Steuber ${ }^{3}$, Stuart L. Weinstein ${ }^{4}$ and Sato Ashida ${ }^{2}$

\begin{abstract}
Background: Research indicates that healthcare providers frequently fail to adequately address patients' health information needs. Therefore, it is not surprising that patients or parents of a sick child are seeking health information on the internet, in particular in online support groups (OSGs). In order to improve our understanding of the unmet health information needs of families dealing with adolescent idiopathic scoliosis (AIS), this study assessed and compared the types of information that adolescents and parents are seeking in OSGs.

Methods: This study used two publicly accessible AIS-related OSGs on the National Scoliosis Foundation (NSF) website that targeted those who are receiving brace treatment and those under observation without treatment. Information exchanges were coded as providing or seeking information. Types of information being exchanged were categorized into several AIS-specific and brace-specific categories. Through a review of over 8,000 messages, 305 adolescents with AIS and 300 parents of a child with AIS were identified and categorized based on stage of illness/treatment. One message from each individual was randomly selected and coded for analysis.

Results: There were significantly more $(p<0.001)$ parents that had a recently diagnosed child compared to recently diagnosed adolescents participating in the AIS-related OSGs, whereas there were significantly more $(p=0.004)$ adolescents that experienced brace treatment compared to parents of a child that experienced brace treatment. The most frequent information exchanged by adolescents and parents was AIS-related concerns regarding causes, diagnosis, and progression of the condition. However, compared to adolescents, parents exchanged this AIS-related information significantly more $(p<.001)$ in their posts. Finally, compared to parents, adolescents exchanged significantly more information about appearance-related concerns regarding both AlS-related deformity $(p<0.002)$ and wearing a brace $(p<0.001)$.

Conclusion: Families dealing with AIS are participating in OSGs to exchange information, in particular information related to the condition and to treatment. This study found similarities and differences regarding how information was exchanged (providing or seeking) and regarding frequency and types of information exchanged. Knowledge of these similarities and differences may be useful for improving health communication in the healthcare setting, at home, and for development and improvement of AlS-related website support.
\end{abstract}

Keywords: Scoliosis, Brace, Spine, Deformity, Internet, Support groups, Adolescent idiopathic scoliosis, Online

\footnotetext{
* Correspondence: traci-schwieger@uiowa.edu

'Department of Biostatistics, The University of lowa, 2400 University Capitol

Centre, lowa City, lowa 52242, USA

Full list of author information is available at the end of the article
} 


\section{Background}

Failure to fully address patients' and families' health information needs in the healthcare setting has frequently been found in the literature regarding a variety of illnesses [1-5]. Therefore, many patients are seeking additional health information outside of the hospital setting, in particular on the internet. For many, participation in online support groups (OSGs) can be a source of illnessrelated information and social support that may help to provide a sense of empowerment through increased acceptance, confidence, optimism, and enhanced social well-being [6-9]. Ultimately, the information and support provided by OSGs may impact several aspects of the patient's illness including, coping with the illness, medical decision-making, behavior changes, preventative behaviors, and expectations.

Research indicates that parents of a child with chronic illnesses are seeking health information in OSGs [10, 11]. Unfortunately, little is known about the use of the internet to obtain health information for adolescents with chronic illnesses and whether the use of the internet as a source for health information differs between adolescents and their parents. One such chronic condition impacting both parents and adolescents is adolescent idiopathic scoliosis (AIS).

Because treatment for AIS includes self-care behaviors, such as wearing a brace and restricting activities, differences in health information acquired (regardless of quality) on the internet by adolescents and parents may result in different understandings, concerns, and expectations regarding the condition and treatments. Ultimately, these differences could result in stress and conflicts in the healthcare setting and at home regarding medical treatment decisions and adherence to treatment recommendations. Therefore, the current study assessed the type of information exchanged by adolescents and parents affected by AIS that are participating in OSGs, including whether the information exchanged was similar or different.

\section{Methods}

The OSGs that were explored in this study were on the non-profit National Scoliosis Foundation (NSF) website where there are more than 8,000 members participating in many different AIS-related forums. This study was part of a larger research project assessing adolescent uncertainty surrounding AIS treatment (observation versus brace treatment), therefore two adolescent NSF forums, "Watching and Waiting" and "Bracing" were analyzed. However, despite the fact that the forums targeted adolescents undergoing observation or brace treatment, many participants were active in both forums simultaneously, discussed both observation and brace treatment, and many were parents of a child with AIS. Therefore, the authors analyzed the type of information exchanged by adolescents compared to parents instead of observation compared to brace treatment. Only posts that were available to the public were analyzed. The first author's institutional review board (IRB) determined this study to be non-human subjects research.

\section{Participants}

Preliminary analysis of more than 8,000 posts was conducted to identify gender, age group (adolescent or parent of a child with AIS), stage of illness/treatment, and topics relevant to the participants. After reviewing all the posts in the two NSF forums, 605 individuals were identified as being either an adolescent with AIS $(n=305)$ or a parent of a child with AIS $(n=300)$. All posts from these two populations were downloaded and each participant's posts were numbered. One post from each participant was randomly selected using a random number generator. The dates of these posts ranged from November 2003 through to August 2014.

\section{Instrumentation}

Categories and subcategories were coded based on the preliminary analysis and establishment of inter-rater reliability. Each information exchange was coded as either providing information or seeking information. Research indicates that the amount and type of illness-related information that an individual seeks is influenced by stage of illness, treatment experience, and consequences of the illness and treatment [12]. Therefore, if mentioned in the post, participants were coded as being recently diagnosed and having undergone (consequences) or currently undergoing common AIS stages of treatment progression (observation, brace, surgery) [13].

The type of information exchanged was coded into three major categories (AIS-specific, brace-specific, and other biomedical information). The AIS-specific subcategories included the following: causes/diagnosis/ progression, co-morbid conditions/pain, functioning, and appearance/deformity. Brace-specific information included the following subcategories: how many hours per day of brace wear (recommended or actual), how long in months or years having undergone or undergoing brace treatment, types of braces, brace non-adherence (mentions of not wearing brace per recommendation), brace effectiveness, physical appearance, brace comfort, daily functioning, exercise/sports, and clothes/dressing. Finally other biomedical information subcategories captured information regarding: doctors/hospitals, and research/resources.

The first author and a graduate student read through randomly selected posts from the two OSGs and recorded the presence of information exchanged regarding the categories and subcategories. The two coders met to 
discuss discrepancies, review decision rules, and combine variable subcategories (if necessary), until acceptable reliabilities were achieved. Acceptable reliability was based on Krippendorff's alpha scores that were greater than 0.80 [14]. After reliabilities were achieved, the two coders independently analyzed $15 \%(n=90)$ of the sample. The inter-coder reliability coefficients for the major information subcategories ranged from 0.87 to 0.97 .

\section{Statistical analysis}

The coding instrument data collection tool was developed using the REDCap data collection software. After the randomly selected posts had been coded, the data was exported and the statistical analyses were conducted using SPSS 21 and SAS Enterprise Guide 4.3. Pearson's Chi-Square was conducted for comparisons between and within the adolescent and parent participants.

\section{Results}

Table 1 describes adolescent and parent characteristics, including stage of illness/treatment, and types of AISrelated, brace-related, and other biomedical information exchanged. As far as stage of illness/treatment there were significantly more parents $(p<0.001)$ participating in the OSGs that had a child that was recently diagnosed with scoliosis compared to adolescents that were recently diagnosed. There were significantly more adolescents participating in the OSGs that had undergone or were currently undergoing brace treatment $(p=0.004)$ compared to parents of a child in this group. Compared to adolescents, parents were exchanging significantly more information regarding doctors/hospitals $(p=<0.001)$.

Table 2 displays most to least frequent of the four AIS-specific and the ten brace-specific information subcategories. Despite information about AIS-related causes, progression, and/or diagnosis being the most frequent information exchange for both adolescents $(n=96)$ and parents $(n=145)$, there were significantly more parents $(p<0.001)$ exchanging (providing and seeking) this information compared to adolescents. Brace-related concerns about appearance was the third most frequent information exchange by adolescents $(n=58)$, which was significantly more $(p<0.001)$ than ninth ranking for parents $(n=26)$. Concerns about AIS-specific deformities and appearance ranked eighth for adolescents $(n=38)$ and was mentioned significantly more $(p=0.002)$ compared to the thirteenth ranked subcategory for parents of a child with AIS $(n=13)$. Finally, while information regarding brace-specific concerns about participation in sports ranked low in frequency in both age groups, parents $(n=17)$ had moderately significantly more mentions $(p=0.034)$ compared to adolescents $(n=7)$.
Table 1 Characteristics of Adolescents with AIS and Parents of a Child with AIS in OSGs

\begin{tabular}{|c|c|c|c|}
\hline & $\begin{array}{l}\text { Adolescents } \\
(n=305)\end{array}$ & $\begin{array}{l}\text { Parents } \\
(n=300)\end{array}$ & $p$ \\
\hline Gender & & & .770 \\
\hline Female & $205(89 \%)$ & $208(92 \%)$ & \\
\hline Male & $25(11 \%)$ & $18(8 \%)$ & \\
\hline \multicolumn{4}{|l|}{ Current/past treatments } \\
\hline Just diagnosed & $28(9 \%)$ & $90(20 \%)$ & $<.001$ \\
\hline Observation & $23(8 \%)$ & $27(9 \%)$ & .515 \\
\hline Brace treatment & $196(64 \%)$ & $158(53 \%)$ & .004 \\
\hline Scheduled for surgery & $14(5 \%)$ & $17(6 \%)$ & .549 \\
\hline Have had surgery & $31(10 \%)$ & $21(7 \%)$ & .165 \\
\hline Type of information exchange & & & .180 \\
\hline Providing information & $160(52 \%)$ & $141(47 \%)$ & \\
\hline Seeking information & $145(48 \%)$ & $159(53 \%)$ & \\
\hline \multicolumn{4}{|l|}{ AIS-specific information } \\
\hline Causes, progression and/or diagnosis & $96(31 \%)$ & $145(48 \%)$ & $<.001$ \\
\hline Co-occurring conditions and/or pain & $45(15 \%)$ & $42(14 \%)$ & .792 \\
\hline Physical functioning & $25(8 \%)$ & $20(20 \%)$ & .474 \\
\hline Appearance & $38(12 \%)$ & $16(5 \%)$ & .002 \\
\hline \multicolumn{4}{|l|}{ Brace-specific information } \\
\hline Daily/nightly brace wear & $40(13 \%)$ & $35(12 \%)$ & .589 \\
\hline Monthly/yearly brace wear & $35(11 \%)$ & $24(8 \%)$ & .150 \\
\hline Types of brace & $62(20 \%)$ & $75(25 \%)$ & .170 \\
\hline Non-adherence to brace wear & $20(7 \%)$ & $13(4 \%)$ & .230 \\
\hline Effectiveness of brace treatment & $52(17 \%)$ & $63(21 \%)$ & .216 \\
\hline Physical appearance in front of others & $58(19 \%)$ & $26(9 \%)$ & $<.001$ \\
\hline Comfortableness of wearing the brace & $57(19 \%)$ & $47(16 \%)$ & .325 \\
\hline Daily physical functioning in brace & $31(8 \%)$ & $27(9 \%)$ & .725 \\
\hline Participation in sports/exercise & $7(2 \%)$ & $17(6 \%)$ & .034 \\
\hline $\begin{array}{l}\text { What to wear/clothes and/or how } \\
\text { to dress }\end{array}$ & $33(11 \%)$ & $27(9 \%)$ & .455 \\
\hline \multicolumn{4}{|l|}{ Other biomedical information } \\
\hline Doctors/hospitals & $14(5 \%)$ & $63(21 \%)$ & $<.001$ \\
\hline Research/resources & $11(4 \%)$ & $16(5 \%)$ & .305 \\
\hline
\end{tabular}

Note. $p$-value is two-sided, significance at 0.05

When comparing message exchanges that were providing information to messages that were seeking information, there were several significant differences within each age group based on stage of diagnosis/treatment, and based on the three information categories (AISrelated, brace-related, and other biomedical). Table 3 summarizes the frequencies and differences in the adolescent and parent groups based on whether the individual was providing information or seeking information. Adolescents were seeking significantly more information than providing it when they were just diagnosed $(p=0.021)$ and 
Table 2 Adolescents' and Parents' AlS-specific and Brace-Specific Information Exchanges by Most to Least Frequently Mentioned Subcategories

\begin{tabular}{|c|c|}
\hline $\begin{array}{c}\text { Adolescents } \\
(\mathrm{n}=305)\end{array}$ & $\begin{array}{l}\text { Parents } \\
(\mathrm{n}=300)\end{array}$ \\
\hline \multicolumn{2}{|c|}{ 1. AIS Causes/Progression $(n=96) \longrightarrow$ 1. AIS Causes/Progression $(n=145)$} \\
\hline 2. Brace Types $(n=62)$ & 2. Brace Types $(n=75)$ \\
\hline 3. Brace Appearance $(n=58)$ & 3. Brace Effectiveness $(n=63)$ \\
\hline 4. Brace Comfort $(n=57)$ & 4. Brace Comfort $(n=47)$ \\
\hline 5. Brace Effectiveness $(\mathrm{n}=52)$ & 5. AIS Co-occur Conditions/Pain $(n=42)$ \\
\hline 6. AIS Co-occur Conditions/Pain $(\mathrm{n}=$ & 6. Brace Daily/Nightly Wear $(\mathrm{n}=35)$ \\
\hline 7. Brace Daily/Nightly Wear $(n=40)$ & 7.5. Brace Dress/Clothes $(n=27)$ \\
\hline 8. AIS Appearance/Deformity $(n=38)$ & 7.5. Brace Daily Function $(n=27)$ \\
\hline 9. Brace Yearly Wear $(\mathrm{n}=35)$ & 9. Brace Appearance $(n=26)$ \\
\hline 10. Brace Dress/Clothes $(n=33)$ & 10. Brace Yearly Wear $(n=24)$ \\
\hline 11. Brace Daily Function $(\mathrm{n}=31)$ & 11. AIS Daily Function $(n=20)$ \\
\hline 12. AIS Daily Function $(n=25)$ & ce Exercise/Sports $(n=17)$ \\
\hline 13. Brace Non-adherence $(n=20)$ & 13. AIS Appearance/Deformity $(n=16)$ \\
\hline 14. Brace Exercise/Sports $(n=7$ & 14. Brace Non-adherence $(n=13)$ \\
\hline
\end{tabular}

Note. $p$-value is two-sided, significance at 0.05

were undergoing observation $(p=0.002)$. Parents of a child that had been recently diagnosed with AIS were also seeking information significantly more than they were providing information $(p<0.001)$. Adolescents that experienced brace treatment were providing significantly more information compared to seeking $(p=0.008)$. Both adolescents and parents of a child were providing information significantly more than they were seeking information when they were scheduled to have surgery $(p=0.011$ and $p=0.012$, respectively) and when they had already had surgery (both groups, $p<0.001$ ).

Adolescents were seeking significantly more than providing AIS-specific information regarding causes/ progression/diagnosis $(p=0.021)$, co-morbid conditions/ pain $(p=0.014)$, daily physical functioning $(p=0.011)$, and appearance/deformity concerns $(p<0.001)$. Adolescents were providing significantly more than seeking brace-specific information regarding daily/nightly brace wear $(p=0.041)$, monthly/yearly brace wear $(p=0.043)$, and effectiveness of brace treatment $(p<0.001)$. Parents were seeking information significantly more compared to providing regarding AIS causes/progression/diagnosis $(p=0.034)$ and co-morbid conditions/pain $(p<0.001)$. Finally, parents were providing information significantly more than seeking information concerning appearance in the brace $(p<0.001)$.

\section{Discussion}

Results from this study indicate that there are similarities regarding the reasons adolescents with AIS and parents of a child with AIS are participating in the AISrelated OSGs. The most sought after information by both adolescents and parents was regarding the causes, progression and/or diagnosis of AIS. Two of the top four most frequent types of information exchanged for both adolescents and parents were regarding types of brace and brace comfort. These findings suggest that, regardless of stage of diagnosis/treatment, adolescents and parents might not be receiving enough information from healthcare providers and are therefore going to the OSGs to exchange (provide and seek) information about other's experiences with the causes, progression and/or diagnosis of AIS and about other's experiences with particular types of braces, in particular the comfort of different braces.

Results from this study also found differences between adolescents and parents participating in the AIS-related OSGs regarding their stage of diagnosis/treatment and regarding the type of AIS-related and brace-related information being exchanged. In general, it appears that parents immediately go online as soon as a child is diagnosed with AIS to seek information regarding the causes of AIS and health conditions associated with AIS. While 
Table 3 Characteristics of Adolescents and Parents Information Exchanges (Seeking or Providing) in OSGs

\begin{tabular}{|c|c|c|c|c|c|c|}
\hline & \multicolumn{3}{|l|}{ Adolescents } & \multicolumn{3}{|l|}{ Parents } \\
\hline & $\begin{array}{l}\text { Providing information } \\
(n=160)\end{array}$ & $\begin{array}{l}\text { Seeking information } \\
(n=145)\end{array}$ & $p$ & $\begin{array}{l}\text { Providing information } \\
(n=141)\end{array}$ & $\begin{array}{l}\text { Seeking information } \\
(n=159)\end{array}$ & $p$ \\
\hline \multicolumn{7}{|l|}{ Past/current treatment } \\
\hline Just diagnosed & 9 & 19 & .021 & 8 & 53 & $<.001$ \\
\hline Observation & 5 & 18 & .002 & 10 & 17 & .278 \\
\hline Brace & 114 & 82 & .008 & 82 & 76 & .073 \\
\hline Having surgery & 12 & 2 & .011 & 13 & 4 & .012 \\
\hline Had surgery & 28 & 3 & $<.001$ & 19 & 2 & $<.001$ \\
\hline \multicolumn{7}{|l|}{ AIS-specific } \\
\hline Causes & 41 & 55 & .021 & 59 & 86 & .034 \\
\hline Co-occurring & 16 & 29 & .014 & 9 & 33 & $<.001$ \\
\hline Functioning & 7 & 18 & .011 & 9 & 11 & .085 \\
\hline Appearance & 10 & 28 & .001 & 12 & 4 & .070 \\
\hline \multicolumn{7}{|l|}{ Brace-specific } \\
\hline Daily/nightly & 27 & 13 & .041 & 19 & 16 & .359 \\
\hline Monthly/yearly & 24 & 11 & .043 & 14 & 10 & .247 \\
\hline Types of & 39 & 23 & .065 & 40 & 35 & .205 \\
\hline Non-adherence & 12 & 8 & .486 & 5 & 8 & .529 \\
\hline Effectiveness & 39 & 13 & $<.001$ & 32 & 31 & .498 \\
\hline Appearance & 36 & 22 & .104 & 21 & 5 & $<.001$ \\
\hline Comfort & 29 & 28 & .791 & 28 & 19 & .060 \\
\hline Functioning & 16 & 15 & .921 & 11 & 16 & .495 \\
\hline Exercise/sports & 4 & 3 & .802 & 7 & 10 & .621 \\
\hline Clothes/dress & 20 & 13 & .322 & 16 & 11 & .182 \\
\hline \multicolumn{7}{|l|}{ Other biomedical } \\
\hline Doctors/hospitals & 7 & 7 & .851 & 23 & 40 & .061 \\
\hline Research/resources & 9 & 2 & .047 & 13 & 3 & .005 \\
\hline
\end{tabular}

Note. $p$-value is two-sided, significance at 0.05

adolescents wait until they have experienced brace treatment to participate in OSGs for the purpose of seeking information regarding AIS-related causes/diagnosis/ progression and for providing information regarding their experiences, in particular how much they wore the brace (per day and years) and whether brace treatment was effective. These findings suggest that, compared to the other, adolescents and parents of a child with AIS might need additional AIS-related information based on their stage of diagnosis and treatment. For example, compared to adolescents, at the onset of AIS diagnosis parents might benefit from additional information from healthcare providers regarding AIS-related information regarding causes/diagnosis/progression, and co-occurring conditions/pain. While adolescents that have undergone or are currently undergoing brace treatment might benefit from additional information from healthcare providers regarding any AIS-related information, in particular causes/diagnosis/progression.
Findings from this study also suggest that adolescents might benefit from additional support and coping strategies regarding other adolescent and parent experiences with AIS-related appearance/deformity concerns, brace wear appearance-related concerns, and regarding relationships between brace wear and brace effectiveness. According to Brashers et al. [13], this type of information and social support can assist in the following ways: facilitate skills development (i.e. giving tips regarding how they changed for PE class with the brace on); giving or receiving acceptance or validation (i.e. validating others frustration regarding brace wear); and encouraging perspective shifts (i.e. telling others that they too were frustrated but wearing the brace was just a short-time in one's life).

In an attempt to provide families affected by AIS with additional information and support that they may be unable to address in the healthcare setting, some healthcare providers are referring families to AIS-related websites 
[15]. While the internet can be a helpful tool for empowering families dealing with AIS, current research indicates that the quality of scoliosis-specific information regarding AIS and treatments is poor, irrelevant, and misleading [16-18]. Findings from this study may provide insight into whether individuals participating in OSGs are negatively or positively biasing information compared to the experiences of individuals that are not participating in the OSGs and compared to AIS-related research findings. For example, adolescents that didn't wear their brace and didn't need surgery might be unintentionally providing support that encourages others to not wear their brace as recommended. On the flip side, adolescents that wore their brace as recommended but still needed to have surgery might be participating in the OSGs to vent their frustration, which may have encouraged others to not adhere to brace wear recommendations.

In summary, healthcare providers need to be aware of how families dealing with AIS are exchanging health information on the internet including: type of format (such as OSGs), who is participating (adolescents and parents), when they are participating (stage of diagnosis/treatment), and the type of and accuracy of information that is being provided. This type of knowledge may help the healthcare provider address any confusion that information from the internet may be generating, in particular information from other adolescents in OSGs regarding their brace-wear experience and whether it was effective. While highlighting the fact that everyone's experience is different, healthcare providers can refer families dealing with AIS to additional quality internet resources for social support and for evidence-based information, such as recent results from the Bracing in Adolescent Idiopathic Scoliosis Trial (BrAIST).

BrAIST research findings indicate that bracing significantly decreased the progression of high-risk curves to the threshold of needing surgery $\left(>50^{\circ}\right.$ largest Cobb angle) in adolescents with AIS [19]. In addition, BrAIST findings indicate that physical appearance and quality-oflife are not negatively impacted by nor does it negatively impact, brace wear compared to individuals undergoing observation or brace wear adherence [20, 21]. BrAIST results, along with the nature and complexity of the condition of AIS and its treatment, can be communicated to families dealing with AIS to help address some of the information, questions, and possibly misinformation that are being generated in AIS-related OSGs.

Because AIS is a less-common condition, for some adolescents and parents, the internet is the only place they may connect with other individuals who have similar experiences and obtain information regarding AIS that is relevant to their lives. Findings from this study highlight the potential for providing web-based support to address the types of information needs and support of families dealing with AIS. Furthermore, findings from this study suggest that the web-based support needs to include information that is tailored to adolescents and parents, to age group and to stages of diagnosis/illness, and provide accurate, evidenced-based information. Ultimately, this type of information and social supportive intervention may improve health communication within and outside of the healthcare setting.

\section{Conclusion}

This study is the first to examine types of health information exchanged in OSGs by adolescents with AIS and parents of a child with AIS. Results from this study suggest that adolescents and parents are seeking and providing information regarding the condition of AIS, AIS-treatment, and other biomedical information in OSGs. This study found differences between adolescents and parents in the stages of diagnosis/treatment and in the type of information being exchanged in AIS-related OSGs, including whether individuals are providing or seeking certain types of information. Therefore, information that is being provided to adolescents with AIS and parents of a child with AIS should take into consideration the age of the individual and the stage of diagnosis/ treatments. This study of adolescents' and parents' information exchange in AIS-related OSGs may help inform priorities in health communication among healthcare providers and adolescents with AIS and parents of a child with AIS, and between adolescents and parents that are dealing with AIS.

\section{Abbreviations}

AIS, adolescent idiopathic scoliosis; IRB, institutional review board; NSF, National Scoliosis Foundation; OSG, online support groups

\section{Acknowledgements}

A sincere thank you to Ellen Schafer for assisting with coding for the purpose of establishing inter-rater reliability. This project was supported by The University of lowa RedCap Grant U54TR001013.

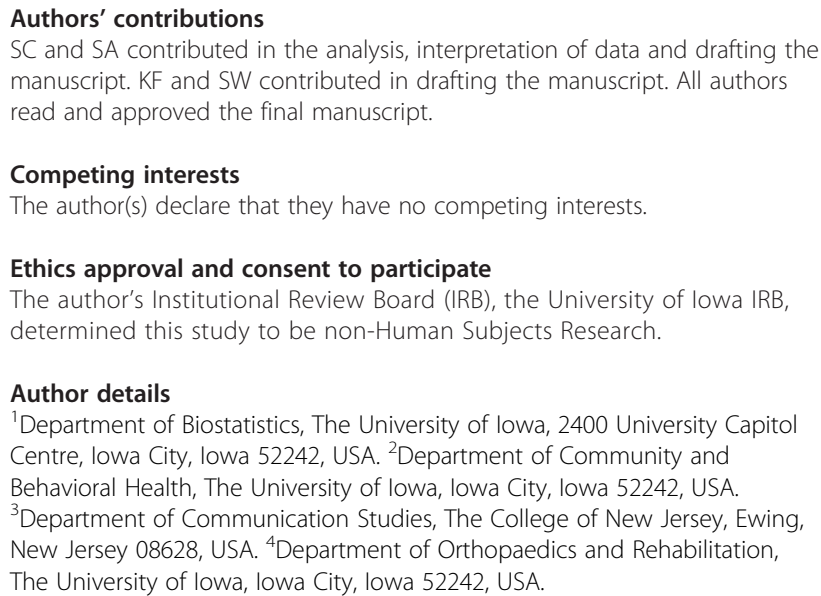

SC and SA contributed in the analysis, interpretation of data and drafting the manuscript. KF and SW contributed in drafting the manuscript. All authors read and approved the final manuscript.

\section{Competing interests}

The author(s) declare that they have no competing interests.

Ethics approval and consent to participate

The author's Institutional Review Board (IRB), the University of lowa IRB, determined this study to be non-Human Subjects Research.

\section{Author details}

${ }^{1}$ Department of Biostatistics, The University of lowa, 2400 University Capitol Centre, lowa City, lowa 52242, USA. ${ }^{2}$ Department of Community and Behavioral Health, The University of lowa, lowa City, lowa 52242, USA ${ }^{3}$ Department of Communication Studies, The College of New Jersey, Ewing, New Jersey 08628, USA. ${ }^{4}$ Department of Orthopaedics and Rehabilitation, The University of lowa, lowa City, lowa 52242, USA.

Received: 13 January 2016 Accepted: 1 August 2016

Published online: 11 August 2016 


\section{References}

1. Iconomou G, Vagenakis AG, Kaolfonos HP. The informational needs, satisfaction with communication, and psychological status of primary caregivers of cancer patients receiving chemotherapy. Support Care Cancer. 2001;9:591-96.

2. Isaksen AS, Thuen F, Hanestad B. Patients with cancer and their close relatives: experiences with treatment, care, and support. Cancer Nurs. 2003;26:68-74.

3. Nordfeldt S, Ängarne-Lindberg T, Nordwall M, Krevers B. Parents of Adolescents with type 1 diabetes-their views on information and communication needs and internet use. A qualitative study. PLoS One. 2013;8:e62096. doi:10.1371/journal.pone.0062096.

4. Sapountzi-Krepia DS, Psychogiou M, Peterson D, Zafiri V, Iordanopoulou E, Michailidou F, Christodoulou A. The experience of brace treatment in children/adolescents with scoliosis. Scoliosis. 2006,l:8. doi:10.1186/1748_7161.

5. Sapountzi-Krepia DS, Valavanis J, Panteleakis GP, Zangana DT, Vlachojiannis PC, Sapkas GS. Perception of body image, happiness and satisfaction in adolescents wearing a Boston brace for scoliosis treatment. Issues Innov Nurs Pract. 2001;35:683-90

6. Davison KP, Pennebaker JW, Dickerson AS. Who talks? The social psychology of illness in support groups. Am Psychol. 2000;55:205-17.

7. Van Uden-Kraan CF, Drossaert CH, Taal E, Shaw BR, Seydel ER, van de Laar MA. Empowering processes and outcomes of participation in online support groups for patients with breast cancer, arthritis, or fibromyalgia. Qual Health Res. 2008;18:405-17.

8. Van Uden-Kraan CF, Drossaert CH, Taal E, Shaw BR, Seydel ER, van de Laar MA. Participation in online patient support groups endorses patients' empowerment. Patient Educ Couns. 2009;74:61-9.

9. Villhauer RP. Perceived benefits of online support groups for women with metastatic breast cancer. Women Health. 2009;49:381-404.

10. Oprescu F, Campo S, Lowe J, Andsager J, Morcuende JA. Online information exchanges for parents of children with a rare health condition: key findings from an online support community. J Med Internet Res. 2013;15(1):e16.

11. Scharer K. Internet social support for parents: the state of science. J Child Adolesc Psychiatr Nurs. 2005;18:26-35.

12. Weinman J, Petrie KJ, Moss-Morris R, Horne R. The illness perception questionnaire: a new method for assessing the cognitive representation of illness. Psychol Health. 1996;11:431-45.

13. Brashers DE, Neidig JL, Goldsmith DJ. Social support and the management of uncertainty for people living with HIV or AIDS. Health Commun. 2004;16:305-31.

14. Krippendorff K. Content analysis: An introduction to its methodology. 2nd ed. Thousand Oaks: Sage Publications Inc:; 2004.

15. Bettany-Saltikov J, Martin D, Wellburn S, et al. Information provided to patients with adolescent idiopathic scoliosis (AIS) at the first point of diagnosis in the hospital clinic: a survey of UK NHS scoliosis consultants. Scoliosis. 2012;7 Suppl 1:036

16. Nason GJ, Baker JF, Byrne DP, et al. Scoliosis-specific information on the internet: has the "information highway" led to better information provision. Spine. 2012;37:E1363-9.

17. Mathur S, Shanti N, Brkaric M, et al. Surfing for scoliosis: the quality of information available on the internet. Spine. 2005:30:2695-700.

18. Wellburn S, Bettany-Saltikov J, van Schaik P. An evaluation of web sites recommended by UK NHS consultant to patients with adolescent idiopathic scoliosis at the first point of diagnosis. Spine. 2013;38(18):1590-4.

19. Weinstein SL, Dolan LA, Wright JG, Dobbs MB. Effects of bracing in adolescents with idiopathic scoliosis. N Engl J Med. 2013;369:1512-21.

20. Schwieger T, Campo S, Weinstein SL, Dolan LA, Ashida S, Steuber KR. Body image and quality-of-life in untreated versus brace-treated females with adolescent idiopathic scoliosis. Spine. 2016;41(4):311-9.

21. Schwieger T, Campo S, Weinstein SL, Dolan LA, Ashida S, Steuber KR: Body image, quality-of-life and brace wear adherence in females with adolescent idiopathic scoliosis. Journal of Pediatric Orthopaedics 2016. (in press).

\section{Submit your next manuscript to BioMed Central and we will help you at every step:}

- We accept pre-submission inquiries

- Our selector tool helps you to find the most relevant journal

- We provide round the clock customer support

- Convenient online submission

- Thorough peer review

- Inclusion in PubMed and all major indexing services

- Maximum visibility for your research

Submit your manuscript at www.biomedcentral.com/submit
) Biomed Central 